\title{
LAS CULTURAS ECONÓMICAS EN EL MUNDO CONTEMPORÁNEO: UN DESAFIO PARA LA UNIVERSIDAD*
}

\author{
Dra. Clara Caselih
}

\section{La naturaleza de la ciencia económica}

Quiero iniciar retomando la definición de economía. ¿En qué pensamos cuando escuchamos esta palabra? "Economías nos evoca la idea de ríqueza, dinero, producción, bienes, servicios, etc

Para entender el sentido más profundo de esta palabra y para entender sus implicaciones culturales se tiene que reflexionar sobre dos aspectos.

Por un lado, la economia tiene que ver con los deseos, las aspiraciones, las necesidades de los hombres. Todos desean algo, pero hay deseos que se pueden satisfacer comprando y usando bienes, que por lo tanto tienen un valor de intercambio, un precio, y se encuentran en un mercado. La economía se ocupa de este tipo de deseos.

Pero, se trata de deseos como todos los demás. Hay dos preguntas interesantes: ¿todos los deseos económicos pueden realizarse? y ¿̇ómo pueden realizarse?

La realidad nos enseña que hay deseos que no se pueden realizar. Por ejemplo, porque es imposible, porque «no tengo tiempok, kno tengo dinero», «no sé cómo hacern, «prefiero hacer otras cosas antes».

Nunca se logra satisfacer de una manera completa todos los deseos: la naturaleza misma del deseo es que es infinito. En la experiencia ueconómica» de

\footnotetext{
Discurso de inauguración del Año Académico 2002
} 


\section{Clara Caselli}

cada uno de nosotros se ve muy bien que cuando logro satisfacer uno de mis deseos, inmediatamente hay otro que surge y me pide satisfacción, y al mismo tiempo me doy cuenta de que cuando consigo algo esperado siempre queda en mí una úluma insatisfacción. Eso pasa exactamente porque los deseos tienen esta característica, que siempre rebotan en algo más, en algo diferente y desconocido. Esto no es económico.

Segundo aspecto. Frente a los deseos en toda su amplitud se necesitan bienes para satisfacerlos. Pero, tcuál es la característica de estos bienes? Que no son infinitos, al contrario, los recursos que se pueden usar para satisfacer las necesidades son escasos. La escasez es la característica principal de los bienes económicos.

El drama de la economia consiste precisamente en esto: que frente a deseos infinitos están recursos escasos $y$, por lo tanto, se tiene que hacet una elección entre los deseos y antes se tiene que establecer una jerarquia entre las necesidades (ivoy a satisfacer primero este deseo y luego este y luego este otro....).

En otras palabras, se trata de escoger una altemativa entre un conjunto de posibilidades de utilización de los tecursos: la economia se constituye como ciencia que estudia la mejor manera para manejar este proceso. Pero es muy evidente que en el establecimiento de prioridades y en la decision de satisfacer un deseo entran en juego elementos que no tienen que ver con la economía, sino con los ideales, los valores, las relaciones que las personas tienen.

\section{Economia y cultura económica}

La vida, entonces, no estă hecha sólo de economia: hay mücho más, Si puedo permitirme parafrasear a Shakespeare: sHay muchas mảs cosas en la tierra y en el cielo de cuantas puede contar tu economian. Nos damos cuenta de esto en la medida que percibimos que no es posible conseguir una satisfacción complera de nuestros deseos y que las necesidades siempre se renuevan, porque siempre aparece un deseo último que está más allá de los aspectos inmediatos e instintivos. Cuando les digo esto a mis alumnos siempre hago este ejemplo para que entiendan: un deseo inmediato es sacar la licenciatura sin trabajar mucho, pero nadie puede decirme que esto es lo que verdaderamente quiere. El deseo más 


\section{LAS CULTURAS ECONŌMICAS EN EL MUNDO CONTEMPORANEO: UN DESAFIO PARA LA UNIVERSIDAD}

verdadero es aprender algo útil para la vida. $\mathrm{Y}_{2}$ al final, ¿no es verdad que hay un deseo más profundo y que se trata del deseo de ser feliz?

Lo que estoy diciendo no es economía en un sentido estrecho, pero tiene que ver con la economia. Cuando los economistas se olvidan de esto y piensan que la idea de hombre económico agota toda la amplitud de la palabra hombre y diseñan estrategias empresariales y politicas económicas con este aliento corto, siempre escogen soluciones que a veces hacen ganar a corto plazo pero no duran en el tiempo, no producen verdadero desarrollo. Si no se toma en cuenta este aspecto, las acciones económicas generan violencia, enriquecimiento, explotación del hombre sobre otro hombre. El desarrollo, al contratio, es algo integral, económico y, al mismo tiempo, social y humano.

Una idea que encuentra mucho consenso entre los economistas es que si todos se preocupan por su propio interés, este comportamiento genera a nivel de la economia un resultado sumamente positivo; el bienestar de todos seria la simple suma de la maximización de las utilidades para cada individuo. En otras palabras, la suma de los egoísmos individuales produciría la satisfacción general. Pero, no es verdad. Se trata de una mentira. Otro ejemplo que les hago a mis alumnos: si hemos preparado un postre muy rico y todos buscan su propio interés habrá alguien que agarra todo sin dejar nada a los demás. Al contrario, puede ser que todos se acuerden que quieren a alguien: en este caso van a comportarse de una manera responsable, es decir, van a compartir el postre con otros (un hermanito, su novia, sus amigos, etc.). En el primer caso, la única manera de poner orden consiste en el hecho de que una persona parta el postre y ponga reglas de conducta para todos. En el segundo caso babrá un desarrollo compartido, un crecimiento del nivel de amistad entre los actores y probablemente también un crecimiento de los recursos: por ejemplo, podría darse que se preparen dos postres o más (hasta llegar a le creación de una pastelería) y, por lo tanto, todos podrian recibir una cantidad más grande.

Esta larga introducción tiene la finalidad de aclarar un punto: la economia no es una ciencia neutral, que prepara soluciones cientificamente perfectas para resolver lo que acabo de llamar el drama de la escasez. Los análisis y las sugerencias que los economistas elaboran están muy estrechamente relacionados con la idea de hombre, de bien individual y de bien común, con los ideales y con los valores que 


\section{Clara Case11i}

están en el trasfondo. Hay algo detrás de la economía que no es simplemente económico. Por eso tiene sentido tratar de identificar las principales culturas económicas que se encuentran en el mundo contemporáneo.

\section{El riesgo como criterio para identificar las culturas económicas}

La diferencia entre las culturas puede ser analizada a través del estudio del comportamiento frente al riesgo, Para entender esto necesitamos retomar la definición de economia como ciencia que estudia las decisiones humanas de elección entre deseos infinitos frente a recursos escasos. Dado que las personas tienen que definit una jeratquia entre los deseos, es evidente que decidir satisfacer un deseo antes que otro implica un riesgo porque luego podriamos darnos cuenta de que nos hemos equivocado y habría sido mejor satisfacer antes otro deseo,

Desde este punto de vista, las mayores culturas que se encuentran en la realidad económica contemporánea son tres:

- Cultura del equilibrio (hombre lógico-racional)

Unos piensan que el riesgo es previsible y puede ser conocido, medido. Ellos opinan que, en efecto, todos los hombres pueden tener acceso a informaciones completas, es decir, saben todo lo que se necesita para prever el riesgo y tomar decisiones lógicas y racionales. Para concluir buenos negocios, lo único que necesitan es un sistema de precios eficiente, el libre mercado, un eficiente mercado del dinera y del crédito (para facilitar el proceso de ahorro y de inversión). No hay ningún imprevisto: nada nuevo puede acontecer. Por ejemplo, hasta hace unos años, en la sociedad japonesa el sólo hecho de nacer en una cietta familia quería decir un recorrido de la existencia ya determinado: desde el tipo de colegio hasta la universidad y el trabajo (un tmbajo para toda la vida, sin cambios y sin riesgos, con avances de carrera preconocidos).

\section{- Cultura de la coordinación (hombre cooperativo)}

Otros piensan, al contrario, que el riesgo no puede ser medido ni previsto porque el ambiente es muy dinámico y en evolución, Si es asi, se trata de buscar la manera de reducirla 


\section{LAS CULTURAS ECONŌMICAS EN EL MUNDO CONTEMPORÁNEO: \\ UN DESAFÍO PARA LA UNIVERSIDAD}

¿Cómo? Una primera alternativa consiste en la disminución del riesgo del mercado a través de acuerdos entre las empresas pata bajar el nivel de competición (creación de oligopolios, es decir, concentraciones de poder y de riqueza en las manos de pocas personas y empresas). Puede tratarse también de acuerdos entre las empresas y sus trabajadores (como en el modelo alemín), entre las empresas y los consumidores, entre las empresas y el estado. En otras palabras, se logra reducir la incertidumbre de la vida económica a través de acuerdos entre los actores (acuerdos, alianzas, pactos). Una segunda alternativa prevé la reducción del riesgo gracias a la intervención del estado, que opera en la vida económica y social llevando los riesgos que las personas y las empresas tendrian que llevar. Merece subrayar que muchas veces se reduce también el nivel de libertad económica (como en el caso de los países socialistas y de los paises que adoptan el modelo del estado de bienestar welfare state).

- Cultura de la competición (hombre competitivo)

También los que se refieren a esta cultura piensan que el riesgo no puede ser medido ni previsto porque el ambiente es muy dinâmico y en evolución, pero ellos están convencidos de que los hombres tienen la capacidad de tomar la incertidumbre como una oportunidad y de luchar para su propia afirmación. La vida económica es una lucha, una competición, y el motot del desartollo es el empresaro individual según decía Schumpeter. En otras palabras, el modelo es un empresano que maneja su empresa cuidando su conveniencia, su interés particular, sin preocuparse mucho de las consecuencias negativas de su conducta en el ambiente social, laboral, en el medio ambiente. Este hombre no tiene relaciones estables con los dernás, porque el criterio con el cual se relaciona es la conveniencia, el interés particular.

En conclusion, se puede decir que hay tres atquetipos fundamentales en el mundo contemporáneo (el hombre lógico-tacional, el hombte cooperativo, el hombre competitivo) y tres culturas económicas mayores (equilibrio, coordinación, competición). Mas o menos estos arquetipos corresponden a la manera de concebir la economia en Japón (pero la cultura japonesa comparte un poco los rasgos del hombre lógico-racional y un poco los del hombre cooperativo), en Europa, en los Estados Unidos. 


\section{Clara Casel11}

Estas culturas económicas están en constante dialèctica entre ellas, con consecuencias muy diferentes sobre la economia mundial Un centro de investigación holandés ha tratado de traducir estas culturas en un sistema de ecuaciones que constituyen un modelo econométrico para medir los resultados que se conseguirian a largo plazo a nivel de la economia mundial en caso de la victoria de cada una de estas culturas.

El hombre competitivo consigue mejores resultados económicos, pero produce desequilibrios sociales a nivel nacional e internacional (por ejemplo, en los Estados Unidos, el sector de la instrucción y el de la salud no son eficientes, y los servicios que brindan no están disponibles para todos; además no hay suficiente atención al problema del medio ambiente y al problema del desarrollo de los paises menos avanzados). El hombre cooperativo, al contrario, cuida los aspectos sociales, pero la reducción del riesgo lo pone menos dinámico y creativo, y conduce a un modelo de economia en el cual el estado representa un papel tan importante que pretende gobernar toda la vida de los hombres. La experiencia de los paises comunistas y la dificultad del proceso de transición a la economía de mercado después de la caida del muro de Berlin han enseñado muy bien que la colectivización de los tiesgos produce la pérdida del sentido de responsabilidad y de la capacidad de emprender. El hombre lógico-racional no deja espacio para el imprevisto, y la persona pierde su creatividad y su identidad individual para anularse en una identidad nacional. Muchas culturas orientales no conocen la idea de kacontecimientom y por eso anulan la personalidad humana en una pertenencia que es dependencia sin libertad: los papás, el esposo, la empresa donde uno trabaja la nación son de hecho los dueños de la vida de los que se relacionan con ellos y toda la vida es concebida en función de un deber abstracto, de algo extraño que no tiene un rostro amigo.

El escenario económico mundial es el terreno en el cual las culturas se enfrentan dialécticamente. Pero, la dialéctica entre ellas to se desarrolla como un debate entre caballeros, sino como un conflicto de intereses económicos y politicos. El resultado siempre es un desastre, porque cada cultura, luchando para su afirmación, subraya aspectos justos, pero parciales, y no puede caer en cuenta de todos los factores que constituyen un hombre. Por eso, todos los éxitos son parciales y no duran en el tiempo. Lo más clamoroso es que, actuando así, los economistas y los políticos hacen prevalecer una idea parcial de la realidad y se 


\section{LAS CULTURAS ECONŌMICAS EN EL MUNDO CONTEMPORÁNEO: UN DESAFIO PARA LA UNIVERSIDAD}

olvidan de buscar cómo la realidad es en verdad. Las consecuencias son muy graves, porque la ideologia, ignorando la realidad, aniquila la libertad del hombre $y$, por lo tanto, no quiere que se vuelva sujeto y protagonista (y elimina también la idea de pueblo, como identidad y pertenencia).

Este aspecto es muy evidente en el debate sobre la globalización. Hace un año lei un artículo muy interesante escrito por Mario Vargas Llosa, que fue publicado con mucho resalte en la prensa europea. Él decia que la globalización es un bien porque favorece la afirmación de la identidad individual, mientras que cualquier identidad de pueblo es enemiga de la libertad. En verdad, él quertía sobre todo criticar los nacionalismos, pero su idea de libertad es con mucha evidencia la libertad de hacer lo que uno quiere y de cortar cualquier tipo de relación. Me parece la idea de globalización que Juan Pablo II muchas veces ha condenado, diciendo que en si la globalización no sería ni un bien ni un mal y que lo que la hace volver un mal, y por lo tanto lo preocupa más, es la ideologia que nace cuando la globalización se vuelve cultura que trata de anular las identidades y las pertenencias. Me parece interesante notar que esto lo preocupa más que la profundización de la distancia cntre los paises ricos y los paises pobres.

\section{¿No hay otra cultura?}

¿Hay otros tipos de hombres económicos?, ¿la cultura cristiana puede sugerir algo diferente? Si observamos la realidad, también en una perspectiva histórica, la respuesta es positiva. No se trata de un modelo descrito en los libros de economía tradicionales, que muchas veces no saben leer roda la realidad. En efecto, en el curso de la historia, los cristianos (y también otros hombres de buena voluntad) han construido iniciativas económicas a partir de su ideal de vida, a partir de su sentido religioso, A veces se trata de iniciativas muy grandes (hospitales, escuelas, bancos para los pobres, etc.) que preceden los estados modernos.

¿Quiènes son los hombres que las han hecho? ¿Cuál es su perfil humano?

El hombre económico que nace de la cultura y de la historia cristiana es un hombre que lucha, que construye, un hombre eprotagonistan, es decir, nin hombre verdaderamente hombre. 
En sintesis tratemos de identificat sus caracteristics:

Antes que nada, la postura frente al céesgo. Él está comprometido con la realidad $y_{s}$ por lo tanto, sabe que el futuro no puede ser conocido ni previsto, especialmente en un mundo complejo como el contemporáneo. Sabe que la perfecta y fría racionalidad económica no existe. Pero la realidad lo solicita, se presenta como oportunidad, desafia su libertad: el imprevisto puede ser positivo. Vale la pena hacer cosas, emprender. Se da cuenta de que hay riesgos y peligros en el camino, pero está dispuesto a correrlos. Arriesgar es necesario para la libertad, dice Luigi Giussani en Edwar es un riesgo. No es el riesgo del jugador, es decir, el riesgo estadístíco, cara o cruz $50 \%$.

Se trata del riesgo de una aventura humana, en que la libertad se desarrolla como capacidad de construir, como deseo de modelar la realidad, también la de la economia, según el ideal de la vida.

A este punto, una pregunta me parece fundamental: ¿para quién arriesgar?, ¿y con quién? Un hombre protagonista - ya lo hemos visto - no puede ser un hombre solo, sin relaciones, cuyo ideal es la afirmación individual. Entonces, si arriesga lo hace porque quiere a alguien y está seguro que alguien lo quiere. Yo siempre cuento la historia del noble caballero de Baune en Borgona que construyó un hospital para los pobres. Él quiso lo mejor para ellos, me impactó mucho cuando lo vi. Y noté una cosa curiosa: sobre las mayólicas, las ollas, los platos, etc. habia hecho poner las iniciales del nombre de su esposa: preguntándome el porqué entendi bien que el deseo de hacer algo útil y el amor para su esposa para él eran la misma cosa.

Por eso, se trata de un hombre que toma riesgos en compañia. Nadie, que esté solo, puede asumir con tranquilidad los riesgos de una iniciativa económica. Se necesita estar abierto a la novedad, con una postura positiva frente a la realidad, pero esto no es posible sin la compañia de otros hombres que puedan dar el coraje y abrir la mirada. Asi el riesgo pierde su carácter negarivo y se vuelve en desafio, en aventura humana. La libertad crece y produce grandes obras adhiriendo a una compañia.

De esta postura nacen unas virtudes económicas que son las que hacen grandes a los empresarios ( $y$ también a las empresas); por ejemplo, la responsabilidad, es decir, la disponibilidad de tomar en cuenta todas las consecuencias de sus acciones, preocupandose por lo tanto por sus trabajadores, por el medio ambiente, 


\section{LAS CULTURAS ECONÓMICAS EN EL MUNDO CONTEMPORÁNEO: \\ UN DESAFIO PARA LA UNIVERSIDAD}

por la camunidad. Y la lealtad: hablamos de un hombre que dice la verdad, que si se compromete cumple su palabra y, por lo tanto, se puede confiar en él. Piensen cómo es importante esta virtud en la economía de la información, en la cual la actividad principal ya no es la transformación de materia prima en productos sino que consiste en la producción y en la circulación de informaciones: las informaciones no son ladrillos o piezas de acero, sino algo que puede ser verdadero o falso (y este es el factor critico de éxito). Otra virtud es la capacidad de vivir errores y fracasos como una oportunidad, porque un hombre no es el resultado de sus errores y además tiene la capacidad de levantarse y volver a empezar. También esta es una virtud muy importante en muestros tiempos: no demorarse mucho para identificar a los culpables y usar el tiempo para remediar los mismos errores hace ahorrar, por un lado, $y$, por otro, permite rapidez y eficiencia.

Ya que no tenernos tiempo para desartollar todas las implicaciones de este discurso, señalo simplemente tres temas.

Primero el tema del pueblo: estamos viviendo en un momento histótico en el cual muchos tienen vergüenza de hablar de pueblo. Cito otra vez Luigi Giussani en el libro Elyo, el poder y las obras. ¿Que es un pueblo? "Gente que de alguna manera se reconoce como amiga y colabora entre si con vistas al ideal de una bumanidad mejor, y que trata de encontrar los instrumentos para poderlo realizan. Una pertenencia verdadera, una identidad, son aspectos fundamentales también para realizar una creatividad económica. No se trata de folklore (muchas veces esta es la caricatura de la identidad, una reducción terrible), se trata de reconocer el recurso más importante para construir.

Segundo. El tema de la política. No solo este tipo de hombre contesta radicalmente la pretensión de la politica de dominar la vida de la gente, sino que pide a la política misma el respeto de la crearividad que nace de la base de la sociedac y exige que el principio furidamental de las relaciones entre el poder $y$ la persona sea la subsidiariedad, como dice la doctrina social de la Iglesia.

Tercero. Otro aspecto interesante es la necesitad de una nueva formulación de la relación entre el estado y el mercado: los fracasos del mercado, asi como los fracasos del estado, se deben a la falta de una cultura económica de protagonismo social. 


\section{Clara Caselił}

\section{Un desafio para la universidad}

Las cosas que acabo de decir son el resultado de reflexiones y la tentativa de encontrar una respuesta a interrogantes que me hago hace muchos años. Yo creo que en mi actual responsabilidad de coordinadora de la Facultad de Ciencias Económicas y Comerciales de esta universidad, yo soy la primera en tener que ponerme en discusión.

El desafio que veo es a dos niveles. Lo digo de una manera muy sencilla: tenemos que formar hombres protagonistas. Esto es lo que el Perú necesita para su desarrollo, esto es lo que el mundo necesita.

Hay tambien otro aspecto: nosotros que somos las autoridades de esta universidad tenemos que tratar de actuar asi. Felizmente, esta es una tarea que comparto con muchos amigos.

En conclusión, lo que he querido proponer en esta charla es la urgencia que haya hombres protagonistas. El tipo humano que he descrito es un constructor, un luchador. ¿Qué construye? Iniciativas, empresas, proyectos, universidades también, que tienen una fuerza ideal, la fuerza de un ideal. La construcción de una iniciativa económica y social es un poco como una sinfonía o un descubrimiento científico. Por un lado es el fruto del genio de una persona, brota de su alma, de su interior; por otro, nace de un encuentro con la realidad, con sus infinitas oportunidades. El hombre protagonista de la construcción económica encuentra la realidad y se hace desafiar, percibe un atractivo en este desafio y decide hacer algo. Hay una primera intuición (la visión) y luego la idea se desarrolla, cambia, se transforma, acaba también. Pero la actividad económica - así como cualquier actividad humana - siempre es asi: de la realidad vienen sugerencias que se tienen que seguir y la genialidad del hombre protagonista consiste en la capacidad de dar forma a la realidad mismá según un ideal, según sus valores y convencimientos, dando su personal contribución a la construcción de un bien común,

Toda la existencia (y su belleza) consiste en esto: seguir siempre, obedecer al camino que la realidad nos propone y a los padres y amigos que Dios nos da en este camino, siempre volviendo a retomar el ideal más grande que nuestro corazón reconoce $y$ al cual se adhiere. 